\title{
OPTICAL METHODS IN CHARACTERIZATION OF HTSC THIN FILM SUBSTRATES
}

\author{
W. RYBa-Romanowski \\ Institute of Low Temperature and Structure Research, Polish Academy of Sciences \\ 50-950 Wrocław, P.O. Box 937, Poland
}

Short overview of optical methods which proved to be useful in the characterization of HTSC thin film substrates is presented. Preliminary tests in polariscopic arrangements, interferometric measurements, optical absorption and emission spectroscopy reveal macroscopic deficiencies of the crystal. Intentionally introduced impurity ions serve as probes of a local strength and symmetry of the crystal field. Results of optical study of $\mathrm{SrLaGaO}_{4}$ and $\mathrm{SrLaAlO}_{4}$ crystals are presented and discussed.

PACS numbers: 78.55.Hx, 42.70.Hj

Performance of high temperature superconducting thin films depends critically on the physical properties of a material used as a substrate. In particular, any potential substrate material should combine an acceptably low dielectric constant and low microwave noise levels with the stable crystalline structure giving minimal mismatch with the superconductor used. In addition to these features, which are inherent to the material considered, a high structural perfection is desired and usually the most difficult to achieve. Methods of optical spectroscopy provide information on both the structural stability and the density of lattice defects which is relevant to the development of high quality HTSC substrates. Among different approaches to get more close insight into the crystal structure of a substrate the behaviour of as grown and intentionally induced structural defects are studied by both the optical absorption and emission spectroscopy in a wide temperature region. In. another approach convenient magnetic ions are introduced to the crystal structure as probes of local crystal field and symmetry. A more sophisticated method consists of an activation of a substrate crystal with ions which are able to sustain the laser action. Considering the information provided by the optical methods we may distinguish macroscopic and microscopic approaches. In the macroscopic approach the data obtained are a veraged values which characterize a certain volume of a sample under study. A microscopic approach is able to give information on the nature of a site occupied by atoms of which the crystal is composed.

In the preliminary macroscopic examination of dielectric material a polariscopic arrangement is useful. In this examination the expanded and collimated light beam from $\mathrm{He}-\mathrm{Ne}$ laser illuminates the sample between crossed polarizers. 

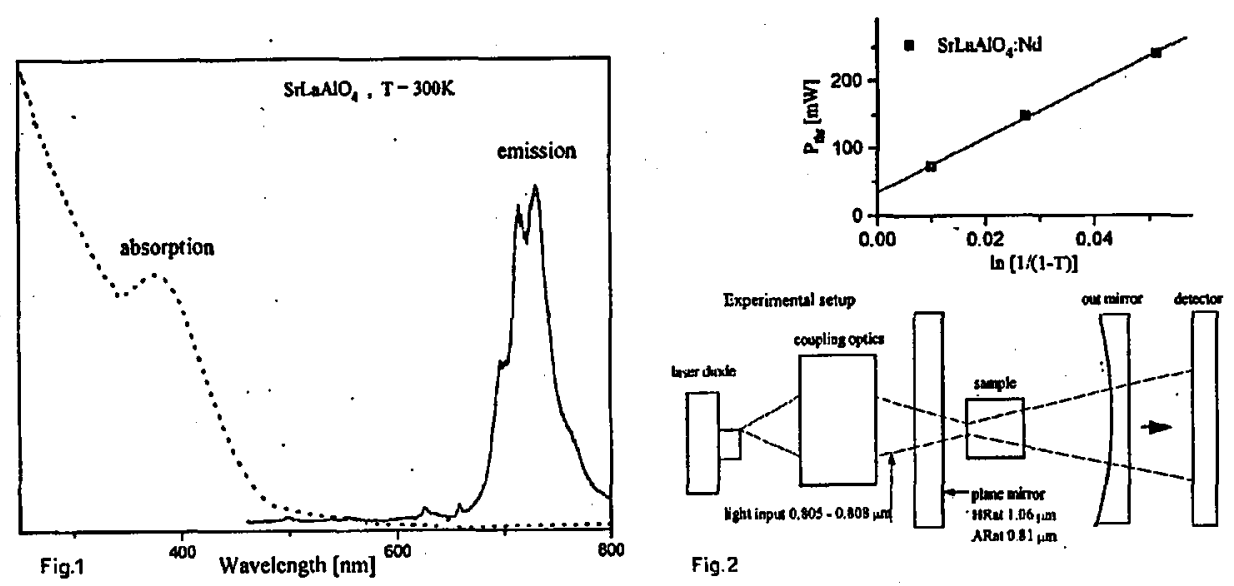

Fig. 2

Fig. 1. Room temperature absorption and emission spectra of point defects in $\mathrm{SrLaAlO}_{4}$ crystals.

Fig. 2. Experimental setup for evaluation of optical losses in crystals by measurements of threshold for stimulated emission as a function of output mirror reflectivity. The inset shows the experimental data for $\mathrm{SrLaAlO}_{4}$ activated with $\mathrm{Nd}$.

Striations and built-in strain give rise to depolarization, the light is partially transmitted by the analyzer and forms the so-called isogyres corresponding to the points of the constant phase difference.

In a more sophisticated method a sample of material under study is inserted in one arm of an interferometer, such as Twyman-Green. Minute inhomogeneities, strain, distortions and inclusions are shown as fringes. In addition to these qualitative tests, which show the macroscopic imperfections, the optical absorption and emission spectroscopy reveals optically active impurities of a material. In Fig. 1 the optical absorption and emission spectra of $\mathrm{SrLaAlO}_{4}$ single crystal is shown. Absorption band stretching from about $500 \mathrm{~nm}$ to the fundamental absorption edge in UV is associated with point defects, presumably $\mathrm{F}$ and $\mathrm{F}^{+}$centres. An emission with a relatively important Stokes shift has been attributed to the $\mathrm{F}^{+}$ centre relaxation. Weak and sharp lines between $500 \mathrm{~nm}$ and $700 \mathrm{~nm}$ have been attributed to traces of $\mathrm{Pr}^{3+}$ impurity. Semiquantitative evaluation of both the passive defects such as strains, inhomogeneities, etc., as well as optically active impurities, involves an intentional introduction of luminescent impurity ions into the crystal structure and an analysis of their stimulated emission. In Fig. 2 we present an arrangement built for an examination of optical quality of $\mathrm{SrLaAlO}_{4}$ and $\mathrm{SrLaGaO}_{4}$ crystals, in which a small amount of La ions was substituted by $\mathrm{Nd}$ ions [1]. A sample in an optical resonator is optically pumped by a diode laser and the threshold for stimulated emission is measured as a function of output mirror reflectivity. The inset in Fig. 2 shows a plot of threshold versus output reflectivity for $\mathrm{SrLaAlO}_{4}$ : Nd "laser quality" crystal having the overall optical losses of $0.02 \mathrm{~cm}^{-1}$. 


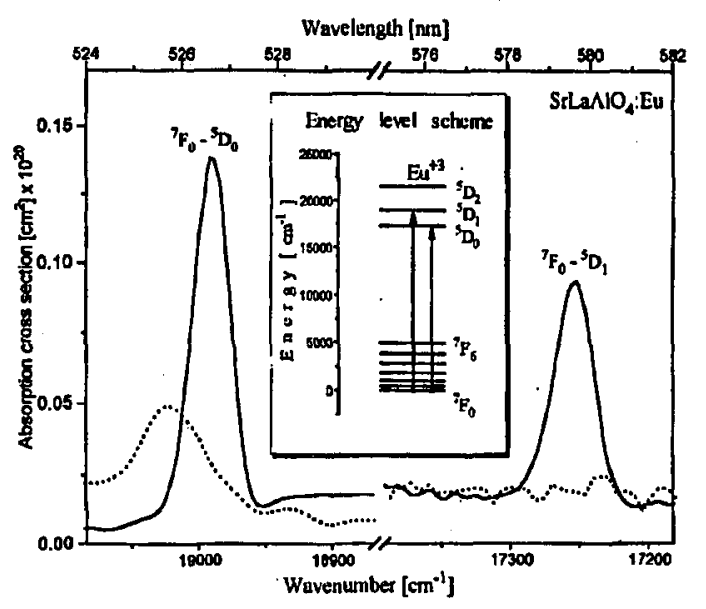

Fig. 3. Room temperature, polarized absorption spectra associated with ${ }^{7} F_{0}-{ }^{5} D_{0}$ and ${ }^{7} F_{0}-{ }^{5} D_{1}$ transitions of $\mathrm{Eu}^{3+}$ in $\mathrm{SrLaAlO}_{4}$ crystal. Solid line $-\pi$ polarization, dotted line $-\sigma$ polarization. The inset shows the relevant energy level scheme for $\mathrm{Eu}^{3+}$.

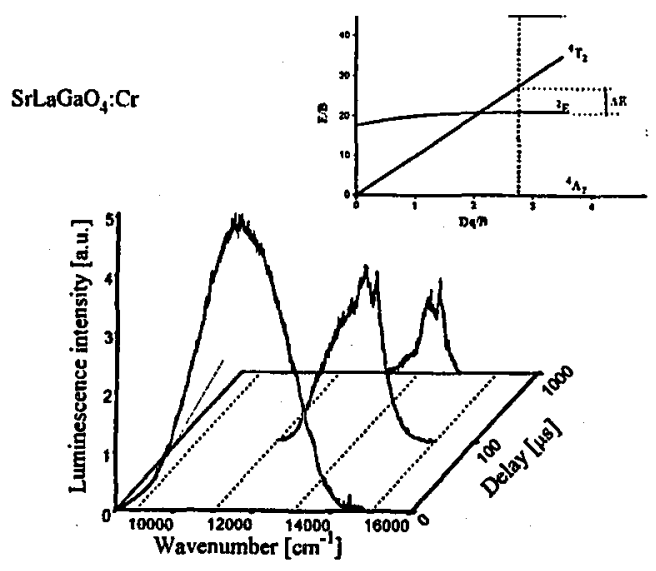

Fig. 4. Time resolved emission spectra of chromium doped $\mathrm{SrLaGaO}_{4}$ crystal showing three different crystal field sites of $\mathrm{Cr}^{3+}$. The inset shows the energies of low lying states as a function of the crystal field strength.

Correlation of optical properties of intentionally introduced impurity ions, with results of X-ray study and ESR measurements provides information on the strength and symmetry of the crystal field acting on atoms of which the crystal is formed. This "microscopic approach" may be useful in the determination of origins of macroscopic imperfections.

The best probes of symmetry and strength of the crystal field are $\mathrm{Eu}^{3+}$ and $\mathrm{Cr}^{3+}$ ions. Trivalent europium with its unsplit ground state and well-isolated 
excited states is relatively easy to deal with. The room temperature, polarized absorption spectra associated with ${ }^{7} F_{0}-{ }^{5} D_{0},{ }^{5} D_{1}$ transitions of $\mathrm{Eu}^{3+}$ in $\mathrm{SrLaAlO}_{4}$ are presented in Fig. 3 together with a relevant energy level scheme indicated in the inset. According to X-ray study $[2,3]$, the structure of the crystal is built up from the layers of $\mathrm{AlO}_{6}$ octahedra formed in the $a b$ plane and between the layers the $\mathrm{Sr}^{2+}$ and $\mathrm{La}^{3+}$ ions are distributed randomly in the sites of $C_{4 v}$ symmetry.

Disparity of size and charge of $\mathrm{Sr}^{2+}$ and $\mathrm{La}^{3+}$ results in certain structural disorder. It can be inferred from the spectra in Fig. 3 that the variation of the crystal field strength gives rise to strong broadening of lines, but the symmetry selection rules for electric and magnetic dipole transitions are fully obeyed indicating that the $C_{4 v}$ symmetry is not distorted.

Unlike the $\mathrm{Eu}^{3+}$ ion, the trivalent chromium provides information in a luminescent study rather than in an absorption measurement. Its optically active electrons belong to the outermost $3 d$ orbitals and are very sensitive to small changes of the crystal field. In Fig. 4 the time resolved luminescence spectra of $\mathrm{SrLaGaO}_{4}: \mathrm{Cr}$ crystal are presented. In the inset the energies of luminescent states of $\mathrm{Cr}^{3+}$ are plotted versus crystal field strength $D q / B$. In the case of the so-called "strong crystal field", where $\Delta E$ value is positive and relatively high, a long lived, narrow band luminescence associated with the spin forbidden ${ }^{2} E-{ }^{4} A_{2}$ transition occurs.

In the weak crystal field $(\Delta E<0)$ a short lived, broad band ${ }^{4} T_{2}-{ }^{4} A_{2}$ emission appears, whereas for $\Delta E$ positive and small the luminescence originates from the ${ }^{4} T_{2}$ and ${ }^{2} E$ levels in thermal equilibrium. Time resolved spectra in Fig. 4 reveal the presence of three $\mathrm{Cr}^{3+}$ sites in a strong, medium and low crystal field, corresponding to three possibilities for the occupancies of the two $A / B$ sites along $\pm c$-axis around each $\mathrm{CrO}_{6}$ octahedron [4]. In the crystals containing abundant point defects the number of different $\mathrm{Cr}^{3+}$ is quite large [5].

\section{References}

[1] W. Ryba-Romanowski, S. Gołąb, I. Sokólska, W.A. Pisarski, G. Dominiak-Dzik, A. Pajączkowska, M. Berkowski, J. Alloys Comp. 217, 263 (1995).

[2] W. Ryba-Romanowski, S. Goląb, J. Hanuza, M. Mączka, A. Pietraszko, M. Berkowski, A. Pajączkowska, J. Phys. Chem. Solids 52, 1043 (1991).

[3] R.D. Shannon, A.A. Oswald, I.B. Parise, B.H.T. Chai, P. Byszewski, A. Pajączkowska, R. Sobolewski, J. Solid State Chem. 98, 90 (1992).

[4] W. Ryba-Romanowski, S. Gołąb, W.A. Pisarski, G. Dominiak-Dzik, A. Pajączkowska, Chem. Phys. Lett. 242, 623 (1995).

[5] W. Ryba-Romanowski, S. Gołąb, A. Gloubokov, A. Pajączkowska, Opt. Mater. 4, 515 (1995). 